\title{
Computational studies of the shear flow behaviour of a model for nematic liquid crystalline polymers
}

\section{$\begin{array}{lll}\text { D. H. Klein } & \text { C. Garcia-Cervera* } & \text { H. D. Ceniceros* }\end{array}$ L. G. Leal ${ }^{\dagger}$}

(Received 4 November 2004, revised 6 April 2005)

\begin{abstract}
We present an accurate and efficient numerical method to the solve the microstructure-flow problem that constitutes the coupling of the molecular-based Doi-Marrucci-Greco model to the Cauchy equation of motion and continuity equation. We also provide a general introduction to the problem of the flow of complex fluids. Preliminary investigations of the predictive capabilities of the Doi-Marrucci-Greco
\end{abstract}

*Department of Mathematics, University of California, Santa Barbara, California 93106, USA. mailto:cgarcia@math.ucsb.edu and mailto:hdc@math . ucsb . edu

$\dagger$ Department of Chemical Engineering, University of California, Santa Barbara, California 93106, USA. mailto:harley@engr.ucsb.edu and mailto:lg120@engr.ucsb.edu

See http://anziamj.austms.org.au/V46/CTAC2004/Klei for this article, (C) Austral. Mathematical Soc. 2005. Published April 26, 2005. ISSN 1446-8735 
model show that with increasing shear rate, in accordance with both experimental observations and other theoretical predictions, the model exhibits three flow regimes: steady linear shear flow at low shear rate; steady roll cells at intermediate shear rates; and irregular flow and orientation patterns at high shear rates. Given sufficiently high shear rates, the irregular flow structure is accompanied by the formation of \pm 1 and $\pm 1 / 2$ strength disclinations. Furthermore, at shear rates large enough to inhibit the tumbling of the average molecular orientation, the solution no longer contains disclinations or roll cells, but retains some structure in the flow direction that has the visual appearance of stripes.

\section{Contents}

1 Introduction

2 The general problem of the flow of complex fluids

3 Generic theoretical structure of flow problems for complex fluids

4 Nematic liquid crystalline polymers

5 A model for nematic LCPs

$6 \quad$ Numerical solution procedures

7 The behaviour of a real material (experimental observations)

C234

8 Summary of computational results

C235

9 Conclusions 


\section{Introduction}

One of the most interesting and important new areas for computational research is the application of numerical methods to problems involving the flow of complex fluids. In this paper, we summarise recent research in this area that has been developed at UCSB under the sponsorship of an NSF-funded Integrative Graduate Education and Research Traineeship (IGERT) program in the area of Computational Science and Engineering.

We assume that many readers of this paper, and many of those in the audience at the CTAC meeting where this work was presented in an invited talk, will not be familiar with the general problem area. Thus, our paper is organised as follows: first, we will provide an overview of what the general problem is all about, why it is important and why, from a computational point of view, it is interesting and challenging in Sections 2 and 3; then, in Sections $4-7$, we discuss the governing equations and computational developments for a specific example problem concerned with the motion of liquid crystalline polymers in a simple shear device; and finally we summarise the results we have obtained to date in Section 8. Once our work on the problem has been completed, a more comprehensive presentation of results will then be forthcoming in a separate paper.

\section{The general problem of the flow of complex fluids}

The generic name "complex fluids" was coined relatively recently in the physics community, though research involving this class of materials has 
been carried out for many years, not only in physics, but to an even greater extent in the engineering and materials communities under such titles as rheology, polymer physics, and colloid and interface science. It is intended to encompass a broad class of materials that are capable of flow such as polymer solutions and melts, colloidal suspensions, liquid crystalline materials (either small-molecule or polymeric), many surfactant solutions above the critical micelle concentration, emulsions and immiscible blends, foams, and even granular materials and powders (an overview of the structure and rheology of complex fluids can be found in [19]). This class of fluids is distinguished qualitatively from Newtonian fluids in the sense that if a disturbance were to cause the system to be in a non-equilibrium configuration, the relaxation back to an equilibrium micro-structural state occurs on long time scales, from milliseconds to seconds in most cases, whereas the corresponding time scales in fluids that we model as Newtonian are typically on the order of microseconds.

One reason that complex fluids are interesting and important is because they are the precursor for many advanced materials applications. In particular, although the ultimate "product" may be a solid, it is typically produced via some type of processing system that involves flow of the original material as a liquid. Because the relaxation times are so long, when we subject the material to flow, the product of the deformation rate (inversely related to the shear-rate) and the relaxation time will frequently be $\mathcal{O}(1)$ or larger. This means that when the material undergoes a flow, this will often induce large changes in the microstructural state of the material, and thus also large changes in its macroscopic properties. One consequence of this is that complex fluids are typically non-Newtonian, viscoelastic liquids. The viscoelasticity of the fluid may change a processing flow, and will change the stress distribution within such a flow, and either of these changes can lead to instabilities that limit the rate of production. A second consequence is that changes in the properties of the material will occur during the processing steps. This may, in principle, be used to advantage in inducing improvements in some desirable property of the finished product. For example, polymers 
that are sold as "electrically conducting" will typically have a very low conductivity in the equilibrium state, but if the polymer chains are strongly stretched and oriented in a common direction during processing, the result may be a material that has a high enough conductivity to become commercially viable. On the other hand, a processing flow may also sometimes induce undesirable changes in the microstructure and thus too in the macroscopic properties. This occurs, as we explain in more detail later, when a liquid crystalline polymer (LCP) is pushed into a mold.

Although empiricism has served us relatively well in the design and development of systems for processing of complex fluids, a future goal must be to improve or even re-design many processes to minimise the impact of instabilities on processing rates (and material properties), as well as optimising desirable properties of some finished material product. Here empiricism will exhibit rapidly diminishing returns, and there is a need to develop a predictive theoretical basis for making the changes that may contribute to these two objectives. An obvious pre-cursor is that we must be able to predict flows; which is partly an issue of developing effective models for the fluid, and partly an issue of developing numerical methods that enables us to use these models to actually predict the flow behaviour of the material. Note that, in contrast with the established methods of computational fluid dynamics (CFD) for Newtonian fluids, successful theory here requires not only prediction of the pressure and velocity fields, but also the coupled changes in the material microstructure. Also note that the development of material models and computational methods are strongly coupled. The governing equations for almost all of the complex fluid models are nonlinear, and the only way of comparing predictions with experimental observations (in all but the simplest rheometer flows) is to use numerical methods to solve the resulting theoretical problem.

It is the objective of our IGERT group on complex fluids to contribute to the development of effective methods and models for the prediction of complex fluid flows. 


\section{Generic theoretical structure of flow problems for complex fluids}

Although the details of the theoretical models are different for all of the different types of complex fluids, there is a basic generic structure that applies to all of them. This provides a convenient framework to discuss some of the qualitative issues associated with the solution of flow problems. The fact that such a framework exists also suggests that progress in the development of numerical methods for one specific type of material may carry over to the complete class of complex fluids.

Historically, the formulation of governing equations was focused largely on the case of polymeric liquids, with constitutive equations for the stress derived via the general framework of continuum mechanics in the form of relationships between the stress and the macroscopic velocity gradient. Partly because this process was not very successful, but also because it became increasingly obvious that it was important to predict not only the velocity, pressure, and stress, but also the spatial and temporal evolution of the microstructural state, the emphasis in more recent years has been on the development of dynamical models based upon a mechanical description of the material at the level of the individual polymer molecules, suspended particles, drops etc. depending on the microstructural nature of the fluid.

In the typical microstructural fluid model, the structure of the fluid is specified by a statistical distribution function $\psi(\mathbf{a} ; \mathbf{x}, t)$ where a represents the multidimensional configuration space, which we represent here symbolically as a vector, though it may be a vector, tensor etc. depending upon the complexity of the particular material. For a suspension or solution of rigid rods, as one example, a is simply a unit vector that specifies the orientation of a rod. If the material were a dilute emulsion, on the other hand, a would represent the shape of a drop. If the deformation were small, the shape would be ellipsoidal, and this can be represented by a second order tensor. For all complex fluids other than liquid crystals, and liquid crystalline polymers, 
the equilibrium state of the material is isotropic. This is either because the structure is statistically isotropic, even though the individual components are non-spherical (as in the case of a rigid-rod suspension or solution, where the equilibrium state is one of random orientations), or because the individual elements reach a spherically symmetric state (as in the case of an emulsion of immiscible drops, or a dilute solution of flexible polymers where the equilibrium state is one of random coils). In the case of liquid crystals (rigid rods with a nematic, that is non-isotropic, potential), the equilibrium state is non-isotropic with a statistically uniform orientation as a function of spatial position, and a distribution around this orientation that represents a balance between rotational diffusion and the nematic alignment potential.

The distribution function changes in flow via a competition between flow-induced deformation away from the equilibrium state, and relaxation (via Brownian motion or other mechanisms) back toward equilibrium. This change in $\psi$ is described by the Fokker-Planck equation (a generalised convection-diffusion equation)

$$
\frac{D \psi}{D t}=f(\psi, \nabla \mathbf{v}) .
$$

The right hand side represents the balance between the deformation process due to the velocity gradient $\nabla \mathbf{v}$ and the relaxation process, which produces a time-dependent change in $\psi$ at a given material point (hence we use the convected or material time derivative). This equation for $\psi$ is a multidimensional partial differential equation (the dimensionality depending on the dimensionality of the configuration space) at each material point. Alternatively, the problem for $\psi$ could be represented via a stochastic differential equation for $\dot{\mathbf{a}}$, with $\psi$ determined as an ensemble average.

Macroscopic properties at any material point depend on the microstructural state at that point. However, in virtually all cases, the macroscopic property of interest turns out to depend not on $\psi$ directly, but rather on moments of the distribution function. For example, the macroscopic stress 
typically depends on the second and fourth moments of $\psi$, that is,

$$
\boldsymbol{\sigma}=\boldsymbol{\sigma}(\mathrm{A}, \mathrm{Q}),
$$

where

$$
\mathbf{A}=\langle\mathbf{a a}\rangle=\int \psi \mathbf{a} \mathbf{a} \mathrm{d} \mathbf{a} ; \quad \mathbf{Q}=\langle\mathbf{a a a a}\rangle=\int \psi \mathbf{a a a a} \mathrm{d} \mathbf{a} .
$$

Finally, for a flow problem, we must solve the Cauchy equation of motion and the continuity equation,

$$
\rho \frac{D \mathbf{v}}{D t}=-\nabla p+\nabla \cdot \boldsymbol{\sigma}, \quad \nabla \cdot \mathbf{v}=0,
$$

provided the fluid is approximated as incompressible.

Starting from some initial condition, a symbolic procedure to solve such a flow problem would involve: first solving the multidimensional convection diffusion (Fokker-Planck) equation to determine $\psi$ at a large number of material points; second, calculating the moments of $\psi$ that are needed to determine the stress; third, determining the divergence of the stress $\nabla \cdot \boldsymbol{\sigma}$; and fourth, solving the equations of motion to determine $\mathbf{v}, p$, and thence returning to Step 1 to calculate the distribution function, and so on to steady state. Although this simplistic sequential series of steps is intended only for illustrative purposes, it is clear that this is a very large problem, and since both (1) and (2) are nonlinear in the dependent variables, it is also highly nonlinear. Clearly, the phrase "large number of material points" means that $\psi$ and the moments of $\psi$ must be calculated at enough material points to obtain an accurate approximation for $\nabla \cdot \boldsymbol{\sigma}$. Furthermore, the equations of motion and continuity must be solved with sufficient spatial resolution that accurate estimates of $\nabla \mathbf{v}$ can be achieved for solution of (1). Finally, it is important to note that the differential equation (1) is itself a significantly large problem, being a multidimensional, time-dependent partial differential equation at each material point. 
Because of the fact that the problem for $\psi$ is quite formidable, and also because we actually need to know only the second and fourth moments of $\psi$ rather than $\psi$ itself, it is common practice to attempt to calculate the moments of $\psi$ directly, rather than first calculating $\psi$ and then calculating the moments. For complex fluids, the procedure for obtaining governing equations for the moment tensors is known as the Prager procedure [28]. However, it is really quite straightforward. To obtain an equation for $\mathbf{A}=\langle\mathbf{a a}\rangle$, we simply multiply equation (1) by the dyadic product aa and then integrate over the complete a-space (as in equations (3)). Symbolically,

$$
\int \mathbf{a a} \frac{D \psi}{D t} \mathrm{~d} \mathbf{a}=\int \mathbf{a a} f(\psi, \nabla \mathbf{v}) \mathrm{d} \mathbf{a} \Longrightarrow \frac{D \mathbf{A}}{D t}=f(\mathbf{A}, \mathbf{Q}) .
$$

Although the moment equation is much simpler (a set of coupled oDEs for the components of $\mathbf{A}$ ), we see that this equation is not closed in the sense that the governing equation for the second moment involves the fourth moment. Similarly, if we were to write an equation for the fourth moment, it would involve the sixth moment, and so on. Thus, a closure approximation is needed. Although this step is not completely understood from a mathematical or physical point of view, it has, until now, generally been necessary to proceed with the approximate closure-based problem, rather than trying to solve the full problem (1)-(4). The question of whether the model problem is a useful approximation of the real problem is then partly a question of the physical assumptions that have been made in deriving (1) and (2), and partly a question of whether the closure approximation is good enough. In practice, it is often very difficult to separate these two questions.

In spite of the fundamental issues related to the procedures described above, it is fair to say that quite considerable progress has actually been made over the past 20 years or so, especially in the development of numerical methods to solve the closure based class of problems for dilute polymer solutions (especially the so-called Boger fluids), and to some extent for the other types of complex fluids. Specifically, significant progress has been made in resolving the so-called "high Deborah number problem", which was 
a name adopted by many of the early researchers to describe the fact that all numerical methods of the time (which incorporated the most sophisticated of known methods for Newtonian fluid CFD) failed to converge beyond a Deborah number De of $\mathcal{O}(1)$ for any non-trivial flow problem using essentially any constitutive model for polymeric liquids. A reasonable summary of the current state of the art can be found in the recent book of Owens and Phillips [27]. In spite of this progress, however, much remains to be done. First of all, current computational algorithms are still limited to a range of Deborah numbers that has only been increased by about one order of magnitude (of course this depends on the problem, the model etc). Secondly, the vast majority of work to date has still focused on models that are intended for dilute polymer solutions. Third, though a great deal of attention has been paid to closure approximations, especially for the latter class of problems, this is still a largely unresolved problem.

The work that we will discuss in the remainder of this paper has been done as part of a new IGERT-based project in Scientific Computation at UCSB. In this program, graduate students are working jointly with faculty from at least two departments as their thesis advisors (for example, mathematics and engineering). The general research objectives within the complex fluids part of this activity are:

1. develop efficient solution methods for both the closure approximated model equations and the un-approximated problem (the focus of any one project is a specific type of complex fluid, but the problems are reasonably generic as we have just attempted to illustrate, and so the lessons learnt for one material will carry over to other systems);

2. try to better understand the approximation inherent in the closure assumptions, and develop improved more systematic methods for dealing with this aspect of the modelling problem;

3. utilise numerical solutions to try to better understand the mechanisms of microstructure-flow interactions. 
Of course the "materials" that we study are only mathematical models. However, they are idealised but physically realisable materials, and they are designed to incorporate the key physical mechanisms of the real material. Of course, this remains to be demonstrated by comparison with the behaviour of the real material and this comparison can only be done via comparison between experimental observation and numerical flow calculations.

\section{Nematic liquid crystalline polymers}

The specific subject of this current project is the flow behaviour of liquid crystalline polymers (LCPS). This class of materials can be envisioned as being comprised of anisotropic rod-like molecules, which are liquid-like in the sense that there is no positional order at equilibrium (that is, the centres of mass are randomly scattered), but are crystalline-like in the sense that they do exhibit orientation order at equilibrium with a strong tendency to align in some common direction. The latter is a consequence of excluded volume interactions. There are two types of such materials, one called lyotropic which is a solution that exhibits a transition between isotropic and nematic (nonisotropic) structures when the concentration is increased sufficiently, and the other called thermotropic which are melts and thus exhibit the isotropic to nematic transition at a critical temperature. The distinction between these systems is not important for the current study. Note that real LCPs are often not a single rod-like molecule but will exhibit some flexibility, including most of the systems that have been generated synthetically, which are typically copolymers with rigid mesogenic units separated by flexible spacers. However, the focus of the current study is a model in which the molecules are envisioned as rigid rods, and the qualitative comparisons that we have made are also with real systems where this is at least a qualitatively correct description.

An obvious question is why study the flow properties of LCPs? One mo- 
tivation is that LCPs are a potentially interesting source of light weight, high tensile strength materials, but that to date they have only been processed into fibres (for example, Kevlar produced by DuPont, and spider silk produced by spiders). Why fibres? It is the strong tendency of LCPs to align at the molecular level that is (in part) responsible for their potential as high modulus materials, and the spinning process required to produce fibres tends to enhance this natural tendency for alignment. Unfortunately, all processing flows other than fibre spinning disrupt the natural alignment in a profound way, thus reducing the LCP to a material that is isotropic from a macroscopic point of view, and thus exhibits properties that are similar to other conventional, but much cheaper polymers. For example, experimental observations have shown that if we subject a uniformly aligned sample to a shear flow (as occurs if we pump the fluid into a mold), it develops a series of hydrodynamic and microstructural instabilities that begin with a hydrodynamic instability to roll cells aligned in the flow direction, followed (or accompanied by) the formation of orientational defects that are known as disclinations. The latter proliferate in the flow, and eventually lead to a "polydomain structure" in which alignment is preserved in tiny microdomains but these are randomly oriented, yielding a macroscopically isotropic material. For an overview of the field, we refer the reader to the book by Donald and Windle [7].

The objectives of our theoretical study are to predict and understand structure-flow interactions for the simplest of all possible flow domains, namely the flow between two parallel plane walls one of which is moving in its own plane to create what would be a simple shear flow if this were a Newtonian fluid. In particular, in the present study we attempt to simulate (and understand) the development of disclinations, and ultimately, the proliferation to the polydomain structure (of course, this is a test of both the model that we have chosen to study, as well as our ability to develop an effective computational approach). Finally, assuming that this step is successful, we may look to where this study may ultimately lead. The next step is to try to understand the relationship between disclination formation and flow type. We know from comparing the behaviour in simple shear flow, and in the 
extensional flow associated with fibre spinning, that the tendency to form disclinations definitely depends on the flow type. It is generally presumed that formation of disclinations is a consequence (in some unknown way) of the presence of tumbling. Finally, earlier work in our group has shown that tumbling can be suppressed even if there is only a weak extensional component superposed on a simple shear flow [3], and thus, as an example, it may be possible that rather minor changes in the geometry of a mold could be sufficient to largely suppress disclinations. Thus, the ultimate goal (which we are still far from approaching) is to utilise the knowledge we will gain of the effects of flow type on tumbling to design processing flows that minimise the formation of disclinations (and everything that follows). In view of these long-range objectives, this paper is just a progress report.

\section{$5 \quad$ A model for nematic LCPs}

We idealise LCPs as a suspension (or solution) of axisymmetric rigid-rod shaped Brownian particles with a mean-field interaction between molecules that favours uniform alignment both locally and via the suppression of spatial gradients of alignment. In particular, we adopt the Doi model [5], with a nonlocal interaction potential suggested originally by Marrucci and Greco [26] (we call this the DMG model). A detailed description, including the derivation of an equation for the stress tensor, was given recently by Feng, Sgalari and Leal [12].

The orientation of a single rod is represented in terms of a unit vector, $\mathbf{u}$. Thus the configuration space in this case is two-dimensional, representable as a point on the surface of a unit sphere in terms of an azimuthal angle $\varphi$ and a polar angle $\theta$, and the distribution function specifies the probability of a particular orientation at a point in space and time,

$$
\psi=\psi(\mathbf{u} ; \mathbf{x}, t)=\psi(\varphi, \theta ; \mathbf{x}, t) .
$$


The evolution equation (1) in this case takes the form

$$
\frac{D \psi}{D t}=\mathcal{R} \cdot D_{r}\left(\mathcal{R} \psi+\frac{\psi}{k_{B} T} \mathcal{R} \phi\right)-\mathcal{R} \cdot(\dot{\mathbf{u}} \psi) .
$$

where $\mathcal{R}$ is the rotational operator, $\mathcal{R}=\mathbf{u} \times(\partial / \partial \mathbf{u})$, that corresponds to the gradient operator in rotational diffusion [1], $k_{B}$ is the Boltzmann constant, $T$ is the absolute temperature, $D_{r}$ is the rotational diffusivity, and $\phi$ is the mean-field nematic potential. The first term on the right hand side represents the effect of rotational Brownian diffusion, the second is the nematic term and is specified in terms of a slightly simplified form of the Marrucci-Greco nematic potential function

$$
\phi=-\frac{3 k_{B} T}{2} U\left(\mathbf{A}: \mathbf{u u}+\frac{\ell^{2}}{24} \nabla^{2} \mathbf{A}: \mathbf{u u}\right),
$$

whereas the third term in (7) represents the effect of hydrodynamic rotation of the rod-like particles/molecules. In this term, the angular velocity of the unit vector $\dot{\mathbf{u}}$ is determined by the general equation for rotation of an axisymmetric particle in a general linear flow, that is,

$$
\dot{\mathbf{u}}=\boldsymbol{\Omega} \cdot \mathbf{u}+\lambda(\mathbf{E} \cdot \mathbf{u}-\mathbf{E}: \mathbf{u u u}),
$$

where $\mathbf{E}$ is the rate of strain tensor and $\Omega$ is the vorticity tensor at point $\mathbf{x}(t)$. The parameter $\lambda$ depends on the shape of the particle. For axisymmetric ellipsoids of aspect ratio $r$ this parameter is just $\lambda=\left(r^{2}-1\right) /\left(r^{2}+1\right)$. In the Doi model, it is assumed that $\lambda=1$.

The nematic potential function (8) contains two terms. The first describes the energy penalty associated with alignment of a particular rod away from the mean orientation at a particular point, and is known in the liquid crystalline literature as the Maier-Saupe potential [25] (this term is often called the local elastic effect, and is associated with viscoelastic effects in the fluid). The second term is the energy penalty for the formation of spatial gradients in the DMG model (it is known as the non-local or gradient elasticity effect, 
and introduces Frank elasticity [4] into the model). One special feature of LCPs is that they generally exhibit some preferred direction of alignment at the boundaries of the flow domain. The nematic potential means that this boundary alignment tends to be maintained as we move away from the boundaries. A full specification of the microstructure problem must then include not only the preceding equations, including the vorticity and rate of strain tensors for the flow, but also the so-called "anchoring" condition at the boundaries of the flow domain. Note that there is a characteristic length scale associated with the non-local term in (8), which is a measure of the distance over which gradient elastic effects are felt. In addition, the form of the second term is simplified compared to the original MarrucciGreco theory, essentially adding the assumption of an equal energy penalty for the three forms of orientation gradient, namely bend, splay, and twist. This simplification is expected to have a quantitative rather than qualitative change in the predicted behaviour of the DMG model LCP [30]. Finally, we note that there is one additional (dimensionless) parameter in (8), namely the nematic strength parameter $U$. In a lyotropic system, $U$ increases with increasing concentration, whereas it increases with decreasing temperature in a thermotropic system.

If the flow were known, the equations (7)-(9) are sufficient to determine how the statistical microstructure of the material evolves in time. Indeed, a number of studies have been carried out using this model, or its simpler original Doi form with $\ell=0$, for fixed flows, especially for simple shear flow $[17,22,9,3]$. In fact, even with the flow fixed, the behaviour predicted by these model equations is quite complex, although apparently qualitatively reasonable. There is an isotropic to nematic transition in the absence of flow at $U=U_{\mathrm{I}-\mathrm{N}}$. For values of $U$ that are smaller than this critical value, the model shows dynamics in flow that are qualitatively similar to a Brownian suspension with no nematic interaction. Thus, for example, in a steady simple shear flow, the orientation distribution function oscillates in time immediately after start-up of the flow, but eventually achieves a final steady state distribution of orientations that is time independent [15]. For larger 
values of $U$, on the other hand, the nematic potential favours having the rods all rotate together in the flow, and in this case, the orientation distribution may remain time-dependent representing the collective tendency for all of the rods to rotate together. This phenomenon of collective rotation is known as "tumbling" in the liquid crystalline literature. In fact, the behaviour of the distribution function at a point where the flow is specified depends in quite a sensitive way on the initial conditions. However, if the mean orientation is initially in the plane of a simple shear flow, and $U$ is held fixed, the model exhibits tumbling at modest flow strengths (that is, for De less than a critical value that depends on $U$ in the case $\ell=0$ ), with a transition to a time-independent distribution function (which is known in the LCP literature as "flow aligning") occurring as the shear rate in the flow is increased above some critical value.

In reality, of course, the flow is not fixed but must be determined along with the microstructure of the LCP. Hence, one must solve the equations of motion and continuity,

$$
\nabla^{2} \mathbf{v}-\nabla p+\nabla \cdot \boldsymbol{\sigma}=0, \quad \nabla \cdot \mathbf{v}=0
$$

with the polymer contribution to the stress, represented here by $\boldsymbol{\sigma}$, determined from the microstructural distribution function in a way that is specified below. Note:

1. the equations (10) are written in the natural form for a suspension (or lyotropic LCP), in which the Newtonian contribution is separated from the stress contribution that is directly attributed to LCP;

2. we have written (10), and will write all of the additional equations, in a nondimensionalized form, with gap width across the shear cell as the characteristic length scale, $l_{c}=H$, the characteristic shear rate $\dot{\gamma}=V / H$, and the pressure and stress scaled scaled by $\eta V / H$; and

3. fluid inertia is negligible compared with the viscous stress since the time 
scale for viscous dissipation is much shorter than that for the evolution of the flow (the latter time scale is associated with rotational diffusion).

Feng et al. [12] have shown that the stress for a DMG model LCP is related to the second and fourth moments of the distribution function

$$
\begin{aligned}
\boldsymbol{\sigma}= & \frac{c}{\beta} \mathbf{E}: \mathbf{Q}+\frac{c}{\operatorname{De}}\left[\left(\mathbf{A}-\frac{\delta}{3}\right)+U(\mathbf{A} \cdot \mathbf{A}-\mathbf{A}: \mathbf{Q})\right] \\
& -\frac{1}{S_{\mathrm{eq}}^{2} \mathrm{Er}}\left[\nabla^{2} \mathbf{A} \cdot \mathbf{A}-\mathbf{Q}: \nabla^{2} \mathbf{A}+\frac{\nabla \mathbf{A}:(\nabla \mathbf{A})^{T}-\nabla \nabla \mathbf{A}: \mathbf{A}}{4}\right],
\end{aligned}
$$

where $c=\nu k_{B} T / 2 \eta D_{r}$ is the concentration parameter ( $\nu$ being the number density of rods), $\beta$ is known as the crowdedness parameter [6], and $S_{\text {eq }} \leq 1$ is the equilibrium value of the scalar order parameter. The dimensionless groups De and Er, known as the Deborah number and Ericksen number, respectively, are defined in the following paragraphs.

Finally, although the model specification (7)-(11) is now complete, we seek solutions at this time only via an approximation of this model, with a closure approximation introduced to allow direct calculations in terms of the second moment tensor A instead of the full distribution function $\psi$. First note that the Prager procedure in this case leads from equation (7) to an equation for the second moment tensor $\mathbf{A}$,

$$
\begin{aligned}
\frac{D \mathbf{A}}{D t}= & (\nabla \mathbf{v})^{T} \cdot \mathbf{A}+\mathbf{A} \cdot \nabla \mathbf{v}-\frac{f}{\mathrm{De}}\left(\mathbf{A}-\frac{\boldsymbol{\delta}}{3}\right)+\frac{f U}{\mathrm{De}}(\mathbf{A} \cdot \mathbf{A}-\mathbf{A}: \mathbf{Q}) \\
& +\frac{f}{2 c S_{\mathrm{eq}}^{2} \mathrm{Er}}\left(\nabla^{2} \mathbf{A} \cdot \mathbf{A}+\mathbf{A} \cdot \nabla^{2} \mathbf{A}-2 \nabla^{2} \mathbf{A}: \mathbf{Q}\right),
\end{aligned}
$$

where the function $f=\frac{4}{9}(1-\mathbf{A}: \mathbf{A})^{-2}$, proposed by Doi [5], accounts for increased rotational diffusivity with increased local order. We use the socalled Bingham closure [2] to approximate the terms $\mathbf{A}: \mathbf{Q}$, and $\mathbf{E}: \mathbf{Q}$ that appear in (11) and (12). As we suggested earlier, the whole issue of closure 
approximations is still a research question. The motivation for this particular closure approximation has been spelt out in earlier papers to which we refer the interested reader $[2,10]$. We simply note here that the Bingham closure does not violate any thermodynamic constraints [32]. Furthermore, it is one of the simplest closure approximations that preserve tumbling in the original Doi model with $\lambda=1$.

There are a number of dimensionless parameters in the DMG model. However, the two key dimensionless numbers that characterise the relative strength of the elastic (nematic) contributions relative to the flow strength are the Deborah number

$$
\mathrm{De} \equiv \frac{\dot{\gamma}}{6 D_{r}}
$$

and the Ericksen number

$$
\operatorname{Er} \equiv \frac{\eta V H}{K}\left[=\left(\frac{24 H^{2}}{c U \ell^{2} S_{\mathrm{eq}}^{2}}\right) \operatorname{De}\right]
$$

where $K=\frac{1}{8} \nu k_{B} T U \ell^{2} S_{\text {eq }}^{2}$ is the elastic constant [12]. Since $(H / \ell)^{2} \gg 1$, it follows from the definition (14) that Er $\gg$ De. When $\operatorname{Er} \geq \mathcal{O}(1)$, the flow is strong enough to induce spatial gradients in the mean orientation. In fact, in the limit of finite Er, but $\mathrm{De} \approx 0$, we can approximate the second moment tensor as

$$
\mathbf{A}=S_{\text {eq }}\left(\mathbf{n n}-\frac{\boldsymbol{\delta}}{3}\right)+\frac{\boldsymbol{\delta}}{3},
$$

where $\mathbf{n}$ is known as the "director", and the DMG model reduces to the wellknown Leslie-Ericksen (LE) model [8, 24]. In this case, the director $\mathbf{n}$ is a function of $(\mathbf{x}, t)$ and the material exhibits elasticity (which is known as Frank elasticity in the LE model) but no viscoelasticity. On the other hand, De provides a measure of the strength of viscoelastic effects which occur when the flow is strong enough to produce distortions in the shape of the distribution function. Since Er $\gg$ De, it is clear that one can have flowinduced changes in the mean orientation with no distortion in the shape of 
the distribution function (for example, tumbling in which the mean orientation becomes time-dependent, but the degree of alignment about the mean orientation remains the same as in the equilibrium (no flow) state). The natural problem to study theoretically is to increase Er, holding the ratio of Er and De fixed, since $\ell$ and $U$ may be viewed as material parameters that will not vary with the shear rate. Similarly, the parameters $c$ and $\beta$ will generally be held fixed for the same reason.

\section{$6 \quad$ Numerical solution procedures}

The problem of simulating the motion of the DMG model for LCPs, subject to the closure approximation discussed above and appropriate boundary conditions, is then to solve (12) for some initial specification of the microstructure and flow to determine the second moment of the distribution function at a large number of points (these may either be material points in a Lagrangian formulation, or we may convert the time derivative to an Eulerian formulation with a large number of fixed points in the flow domain); from $\mathbf{A}$ to use (11) to calculate the stress at these same points and thence the divergence of the stress; and then finally solve (10) for the velocity and pressure fields. As indicated above we would like to do this for a wide range of Er values ranging from modest Er with De small, to values of Er that are large enough to reach moderate to large values of the Deborah number.

This problem was initially addressed in our group using methods that were different from those used here [29], with modest success in terms both of the accuracy of the resulting solutions and the range of parameters for which solutions could be achieved. A schematic of the flow domain is presented in Figure 1. In both these initial calculations and in the new calculations that have been carried out to date, two simplifications have been introduced to reduce the size of the numerical problem. One is that we assume that the flow and microstructure are all periodic in the cross-channel direction 




Figure 1: Schematic of the two-dimensional shear flow domain. The flow field is bounded along the spanwise direction by parallel plates separated a distance $H$ and along the wall-normal direction by periodic boundaries separated a distance $W . V$ is the relative velocity between the two plates.

(that is, the $y$-direction), and thus truncate the width of the channel to some finite value. The second is to assume that there are no gradients in the flow direction (that is, the $x$-direction). The flow is fully three-dimensional in the sense that all three velocity components are nonzero, and we consider a completely general form for the distribution function at each point. The assumption of zero gradients in the flow direction means that we can have rollcells in the flow direction (as observed experimentally) and we can also have disclinations aligned in the flow direction (as also inferred in experimental observations), but these will extend indefinitely in the flow direction. This is not only inconsistent with what is observed, but may also change the dynamics in the sense that all disclination lines must be open, rather than closing on themselves to form loops as occurs for certain types of disclinations in the real system. The periodicity assumption has been partially explored in the present work, in the sense that we have considered flow cells of several different widths to assess the effect that this has on the flow structure. The inclusion of gradients in the flow direction greatly increases the size of the 
problem, and we are currently working to implement a parallel version of the present code to allow this assumption to be relaxed (as well as to enable calculations in more complex flow domains).

As is the case with most viscoelastic flow calculations, the fact that large, localised stress gradients (whose locations are not necessarily known a priori) are ubiquitous, even for cases involving somewhat trivial flow geometries, highly-accurate and robust numerical methods are necessary for modelling the flow behaviour of LCPs. The current solution algorithm is based upon the use of spectral collocation of the velocity, pressure and second moment tensor components in the $y$-direction, with derivatives in the $y$-direction evaluated using fast Fourier transforms. Derivatives in the $z$-direction are evaluated using a sixth-order compact finite difference formulation. The evolution equation for the configuration tensor (12) is integrated using the third-order Runge-Kutta TVD (total variational diminishing) scheme proposed by Van der Vorst [31].

The sixth-order compact finite difference stencil, taken from [23], that is used to approximate the first derivative in the interior of the domain is

$$
\alpha f_{j-1}^{\prime}+f_{j}^{\prime}+\alpha f_{j+1}^{\prime}=\frac{b}{4 h}\left(f_{j-2}-f_{j+2}\right)+\frac{a}{2 h}\left(f_{j-1}-f_{j+1}\right),
$$

where $\alpha=1 / 3, a=14 / 9$, and $b=1 / 9$. The prime in this expression and those that follow denotes the derivative with respect to $z$. Evaluation of the first derivatives at the points just inside of the upper and lower boundaries, where $j=2, \mathrm{nz}-1$, requires the use of a three-point stencil. For these points a fourth-order form of equation (16) is used, where $\alpha=1 / 4, a=3 / 2$, and $b=0$. The fourth-order sided-difference equations used for the boundary points $j=1, \mathrm{nz}$ are

$$
\begin{aligned}
& f_{1}^{\prime}+3 f_{2}^{\prime}=\frac{1}{h}\left(-\frac{17}{6} f_{1}+\frac{3}{2} f_{2}+\frac{3}{2} f_{3}-\frac{1}{6} f_{4}\right), \\
& f_{\mathrm{nz}}^{\prime}+3 f_{\mathrm{nz}-1}^{\prime}=-\frac{1}{h}\left(-\frac{17}{6} f_{\mathrm{nz}}+\frac{3}{2} f_{\mathrm{nz}-1}+\frac{3}{2} f_{\mathrm{nz}-2}-\frac{1}{6} f_{\mathrm{nz}-3}\right) .
\end{aligned}
$$


To approximate the second derivative, we use a sixth-order compact finite difference stencil of the form:

$$
\alpha f_{j-1}^{\prime \prime}+f_{j}^{\prime \prime}+\alpha f_{j+1}^{\prime \prime}=\frac{b}{4 h^{2}}\left(f_{j-2}-2 f_{j}+f_{j+2}\right)+\frac{a}{h^{2}}\left(f_{j-1}-2 f_{j}+f_{j+1}\right),
$$

where $\alpha=2 / 11, a=12 / 11$, and $b=3 / 11$. For points $j=2, \mathrm{nz}-1$, we use a fourth-order form of equation (19), where $\alpha=1 / 10, a=6 / 5$, and $b=0$. The third-order sided-difference equations used for the boundary points $j=1, \mathrm{nz}$ are

$$
\begin{aligned}
& f_{1}^{\prime}+11 f_{2}^{\prime}=\frac{1}{h^{2}}\left(13 f_{1}-27 f_{2}+15 f_{3}-f_{4}\right), \\
& f_{\mathrm{nz}}^{\prime}+11 f_{\mathrm{nz}-1}^{\prime}=\frac{1}{h^{2}}\left(13 f_{\mathrm{nz}}-27 f_{\mathrm{nz}-1}+15 f_{\mathrm{nz}-2}-f_{\mathrm{nz}-3}\right) .
\end{aligned}
$$

Both difference formulations require the solution of tridiagonal systems of equations. We solve the resulting linear systems efficiently using the Thomas algorithm [14].

The steady Stokes form of the equations of motion together with the continuity equation is solved using the Uzawa Bi-Conjugate Gradient Stabilised method. The discrete counterparts to equations (10) can be written as

$$
\begin{aligned}
& L \mathbf{v}-G p=-\mathbf{g}, \\
& D \mathbf{v}=0,
\end{aligned}
$$

where $L, G$ and $D$ are discrete analogues of the Laplacian, gradient, and divergence operators, respectively, and $\mathrm{g}$ represents the divergence of the polymeric stress $\boldsymbol{\sigma}$. Given that $L$ is nonsingular for the discretisation used in this study, we multiply equation (22) by $L^{-1}$ and substitute the resulting expression into equation (23) to obtain the following linear system for $p$ :

$$
A p=f,
$$

where $A=D L^{-1} G$ is the Stokes form of the discrete Uzawa operator and $f=D L^{-1} \mathbf{g}$. Note that, as a result of the use of sided-difference equations in evaluating wall-normal derivatives, the linear system for $p$ is nonsymmetric. 
Compute $\mathbf{v}^{(0)}=L^{-1}\left(G p^{(0)}-\mathbf{g}\right)$ for some initial guess $p^{(0)}$ Set $r^{(0)}=D \mathbf{v}^{(0)}$

Choose $\tilde{r}$ (for example, $\tilde{r}=r^{(0)}$ )

for $i=1,2, \ldots$

$\rho_{i-1}=\tilde{r}^{T} r^{(i-1)}$

if $\rho_{i-1}=0$ method fails

if $i=1$

$$
q^{(i)}=r^{(i-1)}
$$

else

$$
\begin{aligned}
& \beta_{i-1}=\left(\rho_{i-1} / \rho_{i-2}\right)\left(\alpha_{i-1} / \omega_{i-1}\right) \\
& q^{(i)}=r^{(i-1)}+\beta_{i-1}\left[q^{(i-1)}-\omega_{i-1} w^{(i-1)}\right]
\end{aligned}
$$

end if

solve $L \mathbf{z}_{1}=G q^{(i)}$

$w^{(i)}=D \mathbf{z}_{1}$

$\alpha_{i}=\rho_{i-1} / \tilde{r}^{T} w^{(i)}$

$s=r^{(i-1)}-\alpha_{i} w^{(i)}$

solve $L \mathbf{z}_{2}=G s$

$t=D \mathbf{z}_{2}$

$\omega_{i}=t^{T} s / t^{T} t$

$p^{(i)}=p^{(i-1)}+\alpha_{i} q^{(i)}+\omega_{i} s$

$\mathbf{v}^{(i)}=\mathbf{v}^{(i-1)}+\alpha_{i} \mathbf{z}_{1}+\omega_{i} \mathbf{z}_{2}$

$r^{(i-1)}=s-\omega_{i} t$

check convergence; continue if necessary

for continuation it is necessary that $\omega_{i} \neq 0$

end

Figure 2: Pseudocode for Uzawa Bi-Conjugate Gradient Stabilised method. 
For Stokes flow, the linear system for $p$ is well-conditioned, that is, the condition number of the discrete Uzawa operator $A$ is $\mathcal{O}(1)$. Given its smooth convergence behaviour and the fact that the method does not require the explicit evaluation of $A^{T}$, we have chosen to solve (24) using the Bi-Conjugate Gradient Stabilised (BiCGSTAB) method [31]. The pseudocode for the Uzawa BicGstaB algorithm, an adaptation of the Uzawa Conjugate Gradient method [16], is presented in Figure 2. Note that in order to satisfy the no-slip condition for the velocity, homogeneous Dirichlet conditions are imposed on $\mathbf{z}_{1}$ and $\mathbf{z}_{2}$. The formulation of the Poisson solver used to determine $\mathbf{z}_{1}$ and $\mathbf{z}_{2}$ is presented below.

The Uzawa BiCGSTAB algorithm requires the solution of Poisson equations for the velocity search directions $\mathbf{z}_{1}$ and $\mathbf{z}_{2}$. Given that both systems are of the same form, we simply discuss the solution of these systems in terms of the variable $\boldsymbol{\zeta}$. We start with the continuous form for the Poisson equation

$$
\nabla^{2} \boldsymbol{\zeta}=\mathbf{f} .
$$

Since we have periodic boundary conditions in the spanwise direction we take the Fourier transform of (25) to obtain

$$
-k^{2} \hat{\boldsymbol{\zeta}}+\hat{\boldsymbol{\zeta}}^{\prime \prime}=\hat{\mathbf{f}}(k, z)
$$

where $k$ is the spanwise wavenumber, and the prime and carat denote the derivative with respect to the $z$-direction and transformed variables, respectively. Thus, for each point in $z$, given by the index $j$, we have

$$
\hat{\boldsymbol{\zeta}}_{j}^{\prime \prime}=k^{2} \hat{\boldsymbol{\zeta}}_{j}+\hat{\mathbf{f}}_{j}
$$

The compact finite difference stencil (19) presented above yields the pentadiagonal system

$$
C \hat{\boldsymbol{\zeta}}_{j-2}+B \hat{\boldsymbol{\zeta}}_{j-1}+A \hat{\boldsymbol{\zeta}}_{j}+B \hat{\boldsymbol{\zeta}}_{j+1}+C \hat{\boldsymbol{\zeta}}_{j+2}=\alpha \hat{\mathbf{f}}_{j-1}+\hat{\mathbf{f}}_{j}+\alpha \hat{\mathbf{f}}_{j+1},
$$

where $A=-(b / 2+2 a) / h^{2}-k^{2}, B=a / h^{2}-\alpha k^{2}$, and $C=b /(2 h)^{2}$. The parameters $a, b$ and $\alpha$ associated with the fourth- and sixth-order approximations are the same as those used in the appropriate forms of equation (19). 
The sixth-order, five-point stencil is used for points $j=3, \ldots, \mathrm{nz}-2$. For the points next to the boundary $(j=2, \mathrm{nz}-1)$, we use the fourth-order, three-point stencil. The pentadiagonal system is solved using the generalised Thomas algorithm [14].

\section{The behaviour of a real material (experimental observations)}

Although there have been many experimental investigations of the behaviour of LCPs in flow, the most comprehensive, in terms of observations over a wide range of shear rates (and thus also a wide range of Ericksen and Deborah numbers) is the study of Larson and Mead [20, 18] in which a nematic liquid crystalline poly ( $\gamma$-benzyl-glutamate) solution was subjected to shear flows in torsional and planar cells.

Larson and Mead [18] observed that this LCP exhibited two distinct regimes when subjected to shear flow, which are now believed to be generic to all LCPs. These two regimes are referred to as the Ericksen number and Deborah number cascades. The Ericksen number cascade corresponds to the regime of shear rates where $\mathrm{Er} \geq \mathcal{O}(1)$ and $\mathrm{De} \ll 1$. It is characterised by instabilities that arise at low shear rates as a consequence of the competition between the viscous stress imposed by the flow and long-range (gradient) elasticity. The result at moderate Ericksen numbers is the formation of roll cells with their axis in the flow direction, followed, as the shear rate is increased, by increasingly complex time-dependent flows, and ultimately, the evolution of disclinations that proliferate with time and lead to a polydomain structure in which the system is only aligned in small microdomains but is isotropic on larger length scales. During this whole series of flow regimes, the Deborah number remains small, though the Ericksen number can achieve quite large values of $\mathcal{O}\left(10^{4}-10^{6}\right)$. 
However, as the shear rate is increased further, the Deborah number reaches $\mathcal{O}(1)$ (and even larger) values, meaning that the time scale of the flow (that is, $\gamma^{-1}$ ) becomes comparable to (or larger than) the relaxation time scale of the polymer, and viscoelastic effects become important. The various transitions that occur once the Deborah number reaches $\mathcal{O}(1)$ values is what Larson and Mead termed the Deborah number cascade. In this regime, as De is increased, the polydomain structure at first becomes increasingly refined, but eventually, at high enough shear rates, viscoelasticity appears to stabilise the system, the complex polydomain structure disappears and there is again the visual appearance of bands (due to modulations in the mean orientation) in the flow direction, followed at higher shear rates by what is apparently a monodomain structure (no birefringence). The stabilisation of the flow is presumably related to the transition from tumbling to flow aligning, which is known to occur for De in the range from 1-10 (depending on the value of the nematic strength $U$ ).

\section{Summary of computational results}

A complete presentation of results that we have obtained in the present study is currently being prepared for publication elsewhere. Here, we briefly and qualitatively summarise these results.

First, we consider the parameter values that have been studied to date. With regard to the fixed parameters, we used the following values: $\beta=200$, $c=100, U=6$, and $\ell / H=8.5 \times 10^{-3}$. The value used for the crowdedness parameter is typical of lyotropic systems. For a discussion of representative values of $c$, we refer the reader to the work of Feng and Leal [11]. In determining the appropriate choice for the nematic strength $U$, we chose a value such that the DMG model exhibited dynamics consistent with those observed of Larson and Mead [21]. Larson and Mead reported that the PBG solution used in their investigations underwent a tumbling-to-wagging transition at 
De $\approx 2$, followed by a wagging-to-flow-aligning transition at $\mathrm{De} \approx 5$. For the case of the unapproximated Doi theory, these transition De values correspond to $U=6[10]$. The nondimensional interaction length scale was chosen such that, for the parameter values presented here, $\mathrm{Er} / \mathrm{De}=10^{3}$. Therefore, for the results presented here, the only parameters varied were Er and De. For comparison to experiments in which the polymer is sheared between two parallel plates, with a fixed separation distance, this is equivalent to changes in the shear rate when the ratio Er/De is held constant.

The present calculations assume no-slip conditions for the velocity components at $z=0,1$, that is, $\mathbf{v}=(z, 0,0)^{T}$. With regard to boundary conditions for the configuration tensor $\mathbf{A}$, the polymer configuration is anchored at the upper and lower boundaries of the domain such that $\mathbf{A}$ remains fixed in its uniaxial equilibrium form (that is, equation (15) with $S_{\text {eq }}$ at the equilibrium value, which can be shown from equation (12), with no flow and $U=6$, to be 0.74454$)$. Initial conditions for the velocity are linear shear flow. A random-phase perturbation, with an $\mathcal{O}\left(10^{-6}\right)$ amplitude, is introduced into the polymer configuration, with an initial orientation along the $y$-axis.

We consider a range of Ericksen numbers from approximately 40 to 11,000 (and thus of Deborah number from 0.04 to 11). In the work of Sgalari et al. [29] it was not possible to obtain solutions down to sufficiently low Ericksen number to provide an unequivocal comparison with the earlier computations of Feng et al. [13] which used the Leslie-Ericksen theory (strictly valid in the limit De $\rightarrow 0$, as discussed earlier). In the present calculations, we were able to demonstrate that the DMG model produces results that closely mimic the qualitative behaviour observed by Larson and Mead, as well as reducing to the LE results of Feng et al. at the lowest Ericksen numbers. In particular, at the lowest Ericksen numbers, there is a transition from steady shear flow to flow with roll cells in the flow direction, with a decrease in the aspect ratio (that is, width to height) with increasing Er that agrees with the measured data of Larson and Mead as well as the LE calculations of Feng et al. This steady roll cell structure persists from $\mathrm{Er} \approx 45$ up to $\mathrm{Er} \approx 250$. 

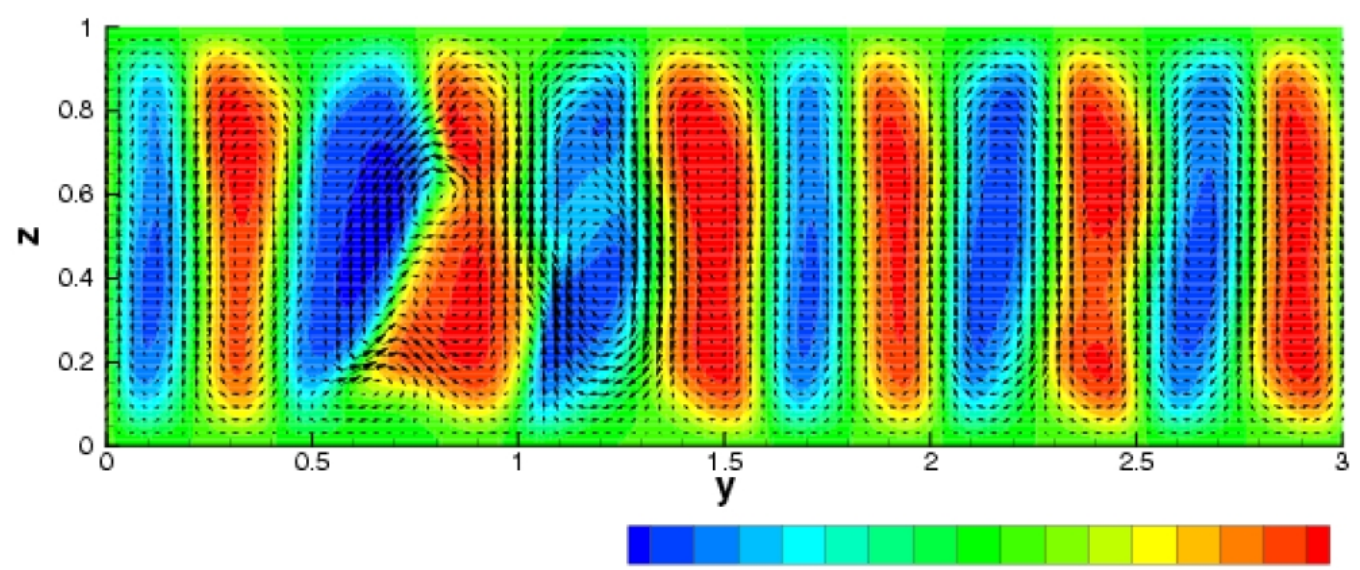

$\theta:-80-70-60-50-40-30-20-10010203040506070$

FiguRE 3: Velocity and director profiles for $\operatorname{Er}=300[\tau=200$ (strain units)]. The vector plot represents the secondary flow components $v_{y}$ and $v_{z}$. The overlying contour plot is the angle $\theta$ between the director and the $y$ - $z$ plane. The relative magnitudes of $v_{y}$ and $v_{z}$, with respect to the shear component $v_{x}$ are $\mathcal{O}\left(10^{-2}\right)$. 
Beyond $\mathrm{Er} \approx 250$, the roll cell structure becomes unstable, and the result is a time dependent quasi-periodic oscillatory flow structure. An instantaneous "snapshot" of this flow in the vorticity-velocity gradient plane is shown in Figure 3. At first, there are no disclinations formed in this rather complex flow, but as the Erickson is increased, the flow continues to become more complex (smaller scale structures that evolve more rapidly) and at $\mathrm{Er} \approx 450$ there is the first appearance of \pm 1 disclinations (where the sign and strength of the disclination denote the relative rotation of the director on a path encircling the disclination core). It is not a surprise to see this type of disclination form. We know that the DMG model reduces to the classical LeslieEricksen model at low De, and we also know from the calculations of Feng et al. that \pm 1 disclinations are seen when this same flow is studied using the Leslie-Ericksen theory. In fact, the \pm 1 disclinations that we see are formed by the same "ridge-splitting" mechanism that was first identified by Feng et al. In the case of the LE model calculations, we only see \pm 1 disclinations because these can escape a true orientational singularity by rotating at their core into the flow direction without any change in the degree of alignment (that is, without any change in the distribution function except for changes in mean orientation). However, the other type of disclination that is commonly seen experimentally, namely $\pm 1 / 2$, is not possible for a pure Leslie-Ericksen fluid because it would produce a true orientational singularity as long as it is not possible to distort the form of the distribution function. Indeed, it is believed that the core of $\pm 1 / 2$ disclinations are actually isotropic, and that local distortion of the distribution function to this isotropic state is the mechanism for avoiding a true singularity in this case. So, it is not surprising that we see only \pm 1 disclinations when the Ericksen number is small enough that the Deborah number is still less than unity. However, the obvious question is whether we begin to see the $\pm 1 / 2$ disclination once the Deborah number approaches $\mathcal{O}(1)$ values where we might expect to begin to see some significant distortions of the distribution function?

The answer is "yes". At Deborah numbers of $\mathcal{O}(1)$ (specifically for Er $\geq 700)$ we begin to see both $\pm 1 / 2$ and \pm 1 disclinations appearing simul- 
taneously in the flow. One interesting question that we will try to answer when enough solutions are available is the relative population of these two families of disclinations, and how this depends on Er (and/or De). Simplistic "energy" arguments based on the energy associated with true $\pm 1 / 2$ and \pm 1 disclinations show that the energy required to form a \pm 1 disclination is four times that for $\pm 1 / 2$ disclinations, and thus we might eventually expect to see a preponderance of $\pm 1 / 2$. However, these arguments do not take into account the actual microstructure in the vicinity of the core for these two types of disclinations, and this presumably negates this whole picture.

Finally, another obvious question is whether the DMG model can reproduce the Deborah number cascade? As we continue to increase Er (and thus De), we encounter an increasingly fine-scale structure and accurate solutions require more and more resolution. In fact, there is an intermediate region between $\mathrm{Er}=1,000$ and $\mathrm{Er} \approx 10,000$ where we have not obtained solutions because we were not willing to bear the extremely high cost with our current code, and non-parallel computing facilities. However, we have obtained a solution at $\mathrm{Er}=11,000(\mathrm{De}=11)$. This is well above the transition in a simple shear flow between tumbling and flow aligning, and we find in the present case that we obtain a solution that is no longer contains disclinations or even role cells. However, it does retain some structure in the flow direction, and this has the visual appearance of stripes, as also seen experimentally in the midst of the Deborah number cascade. At the moment, we have not obtained solutions at either higher or lower values of the Deborah number in this regime.

\section{Conclusions}

We have developed a numerical technique that allows for solutions of the DMG model equations for LCPs for both high and low values of the Ericksen number. By means of this technique, we have been able to demonstrate that 
the DMG model predicts the formation of both \pm 1 and $\pm 1 / 2$ disclinations in a simple shear cell, with the former appearing at lower Ericksen number by a mechanism that is the same as identified earlier by Feng et al. via calculations using the Leslie-Ericksen model, and the latter appearing at higher Ericksen numbers when the Deborah number is $\mathcal{O}(1)$. The model also shows a transition at even higher Ericksen number, when the Deborah number is above the tumbling to flow-aligning transition, to a relatively stable flow with a stripped pattern in the flow direction. Future work will fill in the details of this qualitative picture, and particularly also focus on the development of a parallel version of this code so that computations can be done which allow also for gradients of the flow or microstructure in the flow direction.

Acknowledgment: This work was supported in part by NSF IGERT grant DGE02-21715 and NSF grant DMS-0311911. The authors thank G. Fredrickson, D. Pine, and E. Meiburg for stimulating discussions.

\section{References}

[1] R. B. Bird, C. F. Curtis, R. C. Armstrong, and O. Hassager. Dynamics of polymeric fluids, volume 2: Kinetic theory. John Wiley and Sons, Inc., 2nd edition, 1987. C223

[2] C. V. Chaubal and L. G. Leal. A closure approximation for liquid-crystalline polymer models based on parametric density estimation. Journal of Rheology, 1:177-201, 1998. http://dx.doi.org/10.1122/1.550887 C226, C227

[3] C. V. Chaubal and L. G. Leal. Smoothed particle hydrodynamics techniques for the solution of kinetic theory problems. Part 2: The effect of flow perturbations on the simple shear behaviour of lcps. 
Journal of Non-Newtonian Fluid Mechanics, 82(1):25-55, 1999. http://dx.doi.org/10.1016/S0377-0257(98)00126-8 C222, C224

[4] P. G. de Gennes and J. Prost. The physics of liquid crystals. Oxford University Press, London, 2nd edition, 1993. C224

[5] M. Doi. Molecular dynamics and rheological properties of concentrated solutions of rodlike polymers in isotropic and liquid crystalline phases. Journal of Polymer Science: Polymer Physics Edition, 19:229-243, 1981. http:

//www3.interscience.wiley.com/cgi-bin/abstract/104060267 C222, C226

[6] M. Doi and S. F. Edwards. The Theory of Polymer Dynamics. Oxford University Press, 1986. C226

[7] A. M. Donald and A. H. Windle. Liquid crystalline polymers. Cambridge University Press, Cambridge, 1992. C221

[8] J. L. Ericksen. Anisotropic fluids. Archive for Rational Mechanics and Analysis, 4(3):231-237, 1960. C227

[9] V. Faraoni, M. Grosso, S. Crescitelli, and P. L. Maffettone. The rigid-rod model for nematic polymers: An analysis of the shear flow problem. Journal of Rheology, 43(3):829-843, 1999. http://link.aip.org/link/?JOR/43/829/1 C224

[10] J. Feng, C. V. Chaubal, and L. G. Leal. Closure approximations for the doi theory: Which to use in simulating complex flows of liquid-crystalline polymers? Journal of Rheology, 42(5):1095-1119, 1998. http://link.aip.org/link/?JOR/42/1095/1 C227, C236

[11] J. Feng and L. G. Leal. Pressure-driven channel flows of a model liquid-crystalline polymer. Physics of Fluids, 11(10):2821-2835, 1999. http://link.aip.org/link/?PHF/11/2821/1 C235 
[12] J. Feng, G. Sgalari, and L. G. Leal. A theory for flowing nematic polymers with orientational distortion. Journal of Rheology, 44(5):1085-1101, 2000. http://link.aip.org/link/?JOR/44/1085/1 C222, C226, C227

[13] J. J. Feng, J. Tao, and L. G. Leal. Roll cells and disclinations in sheared nematic polymers. Journal of Fluid Mechanics, 449:179-200, 2001. C236

[14] C. A. J. Fletcher, R. Glowinski, and M. Holt, editors. Computational Techniques for Fluid Dynamics: Fundamental and General Techniques, Vol. 1. Springer-Verlag, New York, 1991. C231, C234

[15] E. J. Hinch and L. G. Leal. Constitutive equations in suspension mechanics. Part 2. Approximate forms for a suspension of rigid particles affected by Brownian rotations. Journal of Fluid Mechanics, 76:187-208, 1976. C224

[16] J. Laminie and U. Meier. Solving Navier-Stokes equations on the Cedar multi-cluster system. Technical Report 978, University of Illinois at Urbana-Champain, Center for Super-Computing Research and Development, 1990. C233

[17] R. G. Larson. Arrested tumbling in shearing flows of liquid crystalline polymers. Macromolecules, 23(17):3983-3992, 1990.

http://pubs.acs.org/cgi-bin/abstract.cgi/mamobx/1990/23/ i17/f-pdf/f_ma00219a020.pdf C224

[18] R. G. Larson. Roll-cell instabilities in shearing flows of nematic polymers. Journal of Rheology, 37(2):175-197, 1992. http://link.aip.org/link/?JOR/37/175/1 C234

[19] R. G. Larson. The Structure and Rheology of Complex Fluids. Oxford University Press, New York, 1998. C213 
[20] R. G. Larson and D. W. Mead. Development of orientation and texture during shearing of liquid-crystalline polymers. Liquid Crystals, 12(5):751-768, 1992. C234

[21] R. G. Larson and D. W. Mead. The Ericksen number and Deborah number cascades in sheared polymeric nematics. Liquid Crystals, 15(2):151-169, 1993. C235

[22] R. G. Larson and H. C. Ottinger. Effect of molecular elasticity on out-of-plane orientations in shearing flows of liquid-crystalline polymers. Macromolecules, 24:6270-6282, 1991.

http://pubs.acs.org/cgi-bin/abstract.cgi/mamobx/1991/24/ i23/f-pdf/f_ma00023a033.pdf C224

[23] S. K. Lele. Compact finite difference schemes with spectral-like resolution. Journal of Computational Physics, 103:16-42, 1992. http://dx.doi.org/10.1016/0021-9991(92)90324-R C230

[24] F. M. Leslie. Some constitutive equations for liquid crystals. Archive for Rational Mechanics and Analysis, 28(4):265-283, 1968. C227

[25] W. Maier and A. Saupe. Eine einfache molekular-statistische theorie der nematischen kristallinflüssigen phase. I. Zeitschrift für Naturforschung Part A - Astrophysik Physik und Physikalische Chemie, 14(10):882-889, 1958. C223

[26] G. Marrucci and F. Greco. The elastic constants of Maier-Saupe rodlike molecule nematics. Molecular Crystals and Liquid Crystals, 206:17-30, 1991. C222

[27] R. G. Owens and T. N. Phillips. Computational Rheology. Imperial College Press, London, 2002. C219

[28] S. Prager. Stress-strain relationship in a suspension of dumbells. Transactions of the Society of Rheology, 1:53-62, 1957. C218 
[29] G. Sgalari, L. G. Leal, and E. Meiburg. Texture evolution of sheared liquid crystalline polymers: Numerical predictions of roll-cells instability, director turbulence, and striped texture with a molecular model. Journal of Rheology, 47(6):1417-1444, 2003. http://link.aip.org/link/?JOR/47/1417/1 C228, C236

[30] J. Tao and J. J. Feng. Effects of elastic anisotropy on the flow and orientation of sheared nematic liquid crystals. Journal of Rheology, 47(4):1051-1070, 2003. http://link. aip.org/link/?JOR/47/1051/2 $\mathrm{C} 224$

[31] H. A. van der Vorst. Bi-CGSTAB: A fast and smoothly converging variant of Bi-CG for the solution of nonsymmetric linear systems. SIAM Journal on Scientific and Statistical Computing, 13(2):631-644, 1992. http://locus.siam.org/SISC/volume-13/art_0913035.html C230, C233

[32] M. van Gurp. Letter to the Editor: On the use of spherical tensors and the maximum entropy method to obtain closure for anisotropic liquids. Journal of Rheology, 42(5):1269-1271, 1998. http://link.aip.org/link/?JOR/42/1269/1 C227 